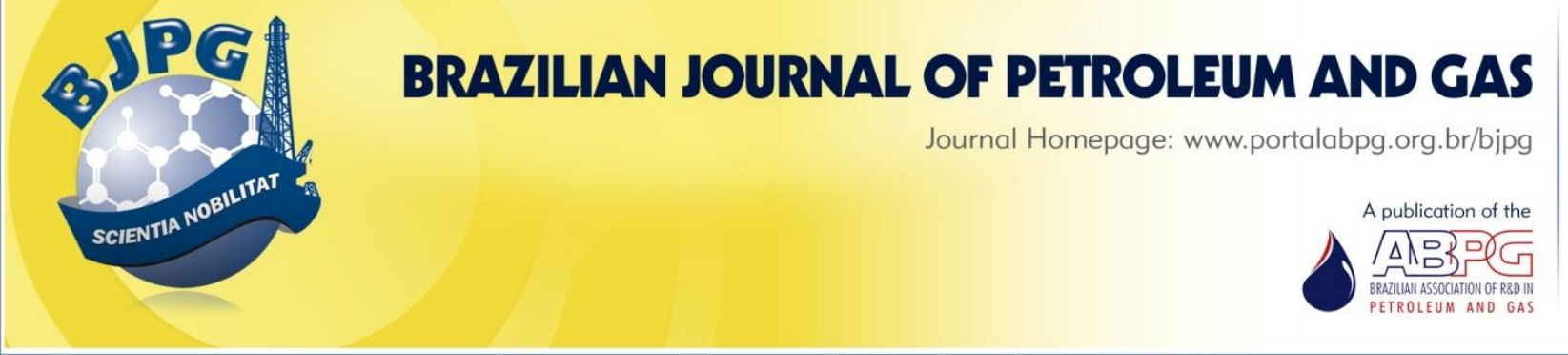

\title{
THE USE OF MATLAB IN THE DETERMINATION OF THERMODYNAMIC PROPERTIES OF NATURAL GAS
}

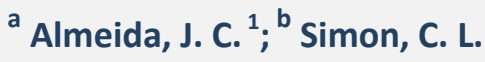 \\ a Natural Gas Company of Paraná- COMPAGAS, Brazil \\ ${ }^{\mathrm{b}}$ Federal University of Paraná- UFPR/PGMEC, Brazil
}

\begin{abstract}
During the preliminary phases of typical operations in the petroleum industry, particularly those involving distribution networks and/or natural gas transmission projects, when complete information is not available, it is sometimes necessary to estimate some thermodynamic properties of natural gas. The behavior of natural gas is affected directly by its chemical composition. It comprises a mixture of volatile hydrocarbons, nitrogen, oxygen, carbon dioxide, and traces of other components. This knowledge is crucial to engineers involved in the areas of equipment design, processing and transportation of such fluids. The design of these systems uses conventional flow equations for both stationary and transient systems, which also include various natural gas fluid properties such as density, gas constant, specific heat, critical temperature and pressure, compressibility factor, and dynamic viscosity, among others. In view of this, a proposition is given to determine the main properties of natural gas from its average chemical composition and related operational conditions (pressure and temperature). This proposal, developed in Matlab, allows the determination of some of the key properties of natural gas, giving users the option to choose the most representative equation of state among the available models, namely, Peng-Robinson, Patel-Teja-Valderrama, Shell Oil Company, Soave-Redlich-Kwong, and van-der-Waals, to determine the compressibility factor of the samples. The results of a particular simulation were compared with actual data from the distribution network COMPAGAS, which is the company responsible for the distribution of piped natural gas in the state of Paraná (Brazil), demonstrating a good convergence between the values obtained and encouraging their use in future.
\end{abstract}

\section{KEYWORDS}

natural Gas; thermodynamic properties; Matlab; compressibility factor

\footnotetext{
${ }^{1}$ To whom all correspondence should be addressed.

Address: Natural Gas Company of Paraná - COMPAGAS, Rua Hasdrúbal Bellegard, 1177 - Cidade Industrial, Curitiba, PR, Brazil Zip Code: 81460-120 | Phone number: (55) 41 3312-1924 | e-mail: j.cezar@compagas.com.br ; j.cezar@ufpr.br doi:10.5419/bjpg2015-0015
} 


\section{INTRODUCTION}

The demand for primary energy is growing continuously. Currently, fossil fuels such as oil, natural gas, and coal produce about $87 \%$ of the primary energy consumed on the planet, according to the website worldwatch. In 2012, the BP Statistical Review of World Energy stated that global natural gas reserves were estimated at 208.4 trillion cubic meters. This number corresponds to about 195 million tons of equivalent oil (considering a daily average consumption of about 8.83 billion cubic meters). This context justifies the prediction that natural gas will gain even more space as a source of available energy in the world. Traditionally, natural gas production is treated as a secondary source of energy, when compared with oil, despite the steady growth in gas consumption promoted by economic, ecological, and technological factors, associated with a growing global demand for energy.

It is an abundant resource that is also environmentally cleaner, if compared to oil and coal, considering that it presents technological advances in the processing, production and transportation. Its main constituent is methane, with fractions of other heavier hydrocarbons such as ethane, propane, and butane, as well as small percentages of other constituents such as nitrogen and carbon dioxide. Variations in such composition, however, may cause relatively significant differences in preliminary calculations when devising distribution network projects and/or transmission pipelines for this fuel. It is known that the typical properties of natural gas will be affected by composition, but more complete information is often either unavailable or difficult to acquire.

This paper seeks to determine the main properties of natural gas (molecular weight, density, gas constant, specific heat, critical temperature and pressure, critical compressibility factor, acentric factor, specific mass, compressibility factor, and dynamic viscosity) from its average chemical composition and corresponding operating conditions (pressure and temperature). This proposal, developed in the Matlab environment, determines these properties allowing users to select the equation of state that more appropriately represents real samples, among some of the models available in the literature (Peng-Robinson, Patel-Teja-Valderrama,
Shell Oil Company, Soave-Redlich-Kwong, and vander-Waals) for determining the compressibility factor. A comparison between the simulations made and real data provided by the COMPAGAS distribution network- a company responsible for the distribution of piped natural gas in the state of Paraná (Brazil), is also discussed, encouraging the implementation of this proposal based on the convergence of values obtained.

\section{Thermodynamic properties of natural gas}

\subsection{Real gas and ideal gas}

Like in the study of other gases, natural gas can be treated either as an ideal gas or as a real gas, depending on the states of interest and the required level of precision. The use of the ideal gas model allows the simplification of the calculations, given the fact that the corresponding state equation is simpler than those that describe a real gas. However, the equations for real gases have the advantage of being valid for a broader range of situations and, in general, one can say that they are more accurate. Therefore, it is appropriate to say that equations of state for real gases are generally more complex mathematical expressions and end up negatively affecting computational time. According Borges (2009a), equations of state for natural gas can be obtained through relationships that are valid for homogeneous substances, using the principle of corresponding states. This is equivalent to consider natural gas as a pure pseudo substance. This view is reinforced by the fact that it is a mixture of hydrocarbons (paraffinic), having methane as its major component. For these reasons, analyses performed considering natural gas as a pure substance usually produce satisfactory results.

The gas is considered "ideal" when all thermodynamic relationships developed with the perfect gas model are supposed to be valid for any temperature and pressure. In practice, however, this proposition is only valid for low pressures, although there is a possibility of making a relation of the properties of a real gas in any state with a real gas in a default state. The properties of the gas may be obtained directly from laboratory measurements or from calculations related to their 


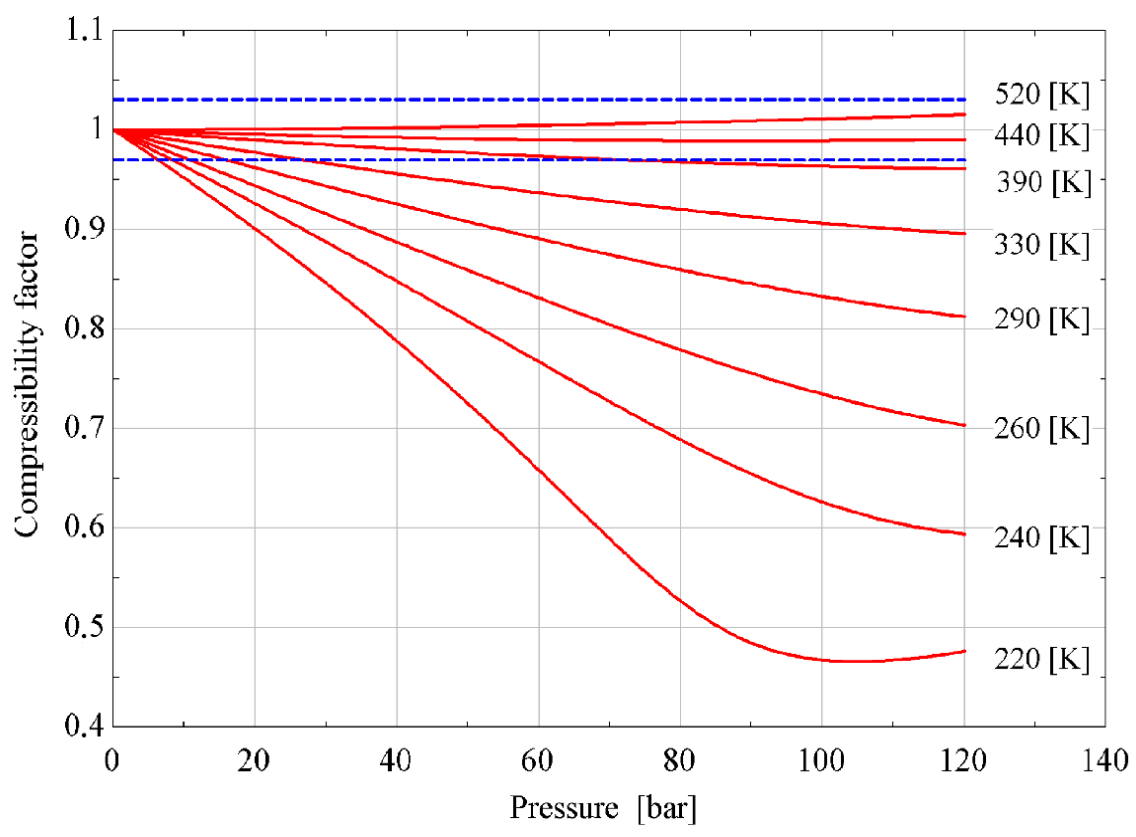

Figure 1. Compressibility factor for methane (Almeida \& Velasquez, 2013).

chemical composition. Such calculations are based either on physical properties of the individual components of the mixture or on rules that relate to the properties of the entire mixture.

On the other hand, the ideal gas model may not be suitable to represent the behavior of a real gas. Therefore, it is essential to know the extent that the behavior of a real gas deviates from a gas considered ideal in a particular state, because such deviation depends on pressure, temperature, and composition. This estimation can be made with the compressibility factor (Z), which can be inserted in the basic ideal gas equation of state, as presented in Equation (1). Obviously, Z = 1 for an ideal gas.

$p v=Z R T$

\subsection{Compressibility factor and equations of state}

The compressibility factor of a gas depends on its temperature and pressure, and more and more intense deviations from the ideal behavior occur as $Z$ deviates from unity $(Z>1$, or $0<Z<1)$. Thus, the ideal gas model could only be used to adequately represent the behavior of the gas corresponding to states for which the $Z$ values are close to unity, within a tolerance range. For example, assuming that this range includes values between 0.97 and 1.03 , the states for which the ideal gas model could be used to represent the behavior of methane are those highlighted in Figure 1, within the blue dashed lines. It is noted that, at a room temperature of $290 \mathrm{~K}$, methane can only be treated as an ideal gas at pressures below 14 bar, since the tolerance range will not be obeyed for higher pressures at this temperature, and therefore the ideal gas model would become inadequate.

The experimental determination of the $Z$ values is relatively expensive and time consuming. Alternatively, it is possible to adopt the use of charts or tables obtained from pure gases for experimental studies, which however may have differences due to the accuracy of their own values, for the compressibility factor, pressure, and temperature. Finally, there are the so-called empirical correlations, models based on the principle of corresponding states, the so-called state equations, which are formulated with the aid of the principle of the corresponding states proposed by van-der-Waals (Kumar, 2004), which establishes universal relationships involving pressure, temperature and specific volume with their corresponding critical parameters, as indicated by Equation (2): 
$p_{R}=p / p_{c}$

$T_{R}=T / T_{c}$

$v_{R}=v / v_{c}$

Where the subscripts $R$ and $C$ indicate reduced and critical properties, respectively. An important consequence of the principle of corresponding states lies in the fact that different gases, which are in states characterized with the same reduced properties, will have the same departure from the ideal gas behavior. This concept is even more reasonable for gas mixtures that exhibit similar molecules in their chemical nature, shape and size, which is typical of natural gas. In spite of being initially proposed for pure gases, the principle of corresponding states has been extended by Kay (1936) for gas mixtures by introducing the concept of additional pseudocritical properties, defined as follows:

$T_{p c}=\sum_{i} y_{i} T_{p c i}$

$P_{p c}=\sum_{i} y_{i} P_{p c i}$

Where $y_{i}$ is the mole fraction of component $i$. Reduced properties defined on the basis of pseudocritical values are often referred to as pseudoreduced properties. Borges (2009b) also points out that many studies with satisfactory results on the compressibility factor of gases, even with different compositions, corroborate the concept of pseudoreduced pressure and temperature.

The literature abounds on the number of models that can be used to calculate the compressibility factor. Generally, these models are classified according to the complexity of the mathematical expression that they represent, and may be more or less accurate (Chvidchenko, 2008). In this study, the ideal gas model was compared with the models of Peng-Robinson, Patel-TejaValderrama, Shell Oil Company, Soave-RedlichKwong, and van-der-Waals, which are presented in Table 1 as a function of reduced temperature and reduced pressure (Almeida \& Velasquez, 2013; Borges, 2009a; Chvidchenko, 2008; Kumar, 2004; Rabah \& Mohamed, 2010). It should be noted that the models of Peng-Robinson and Soave-Redlich-
Kwong use the acentric factor of gas $(\omega)$, whilst the Patel-Teja-Valderrama model requires the critical compressibility factor $\left(Z_{c}\right)$. In the case of a gas mixture, these parameters are determined by the following expressions:

$\omega=\sum_{i} y_{i} \omega_{i}$

$Z_{c}=\sum_{i} y_{i} Z_{c i}$

The acentric factor gas is valid to describe deviations in the behavior of a fluid with regard to the non-spherical shape of the molecules. Many abacuses and tables of chemical properties of substances show their respective values (Peixoto, 2005/2006).

\subsection{Dynamic viscosity of natural gas}

The motion of a Newtonian fluid can be characterized by the continuous deformation when subjected to shear stress, which is proportional to its strain rate, and can be represented by:

$\tau_{W}=\mu \frac{d \mathrm{v}}{d y}$

Where the proportionality constant corresponds to the absolute viscosity of the fluid $(\mu)$. In the case of gases, $\mu$ increases with pressure and decreases with temperature.

Several procedures for determining the dynamic viscosity of natural gas are available. The empirical equation given by the API-65 PROJECT is considered a viable option, given the fact that it provides reasonable accuracy with certain mathematical ease. See Equation 8:

$$
\mu=10^{-4}\left[\frac{(10.425+0.244 d) T^{1.5}}{68.02+208.4 d+T}\right] e^{(X \rho Y)}
$$

Equations 9 and 10 correlate the empirical factors $X$ and $Y$ :

$X=2.576+\frac{1063.58}{T}+0.276 d$

$Y=1.108+0.0404 X$

These equations take into account the density of the fluid $\left(\mathrm{g} / \mathrm{cm}^{3}\right)$, the absolute temperature $(K)$, and the corresponding dynamic viscosity (cp). 
Table 1. Models proposed to calculate the compressibility factor.

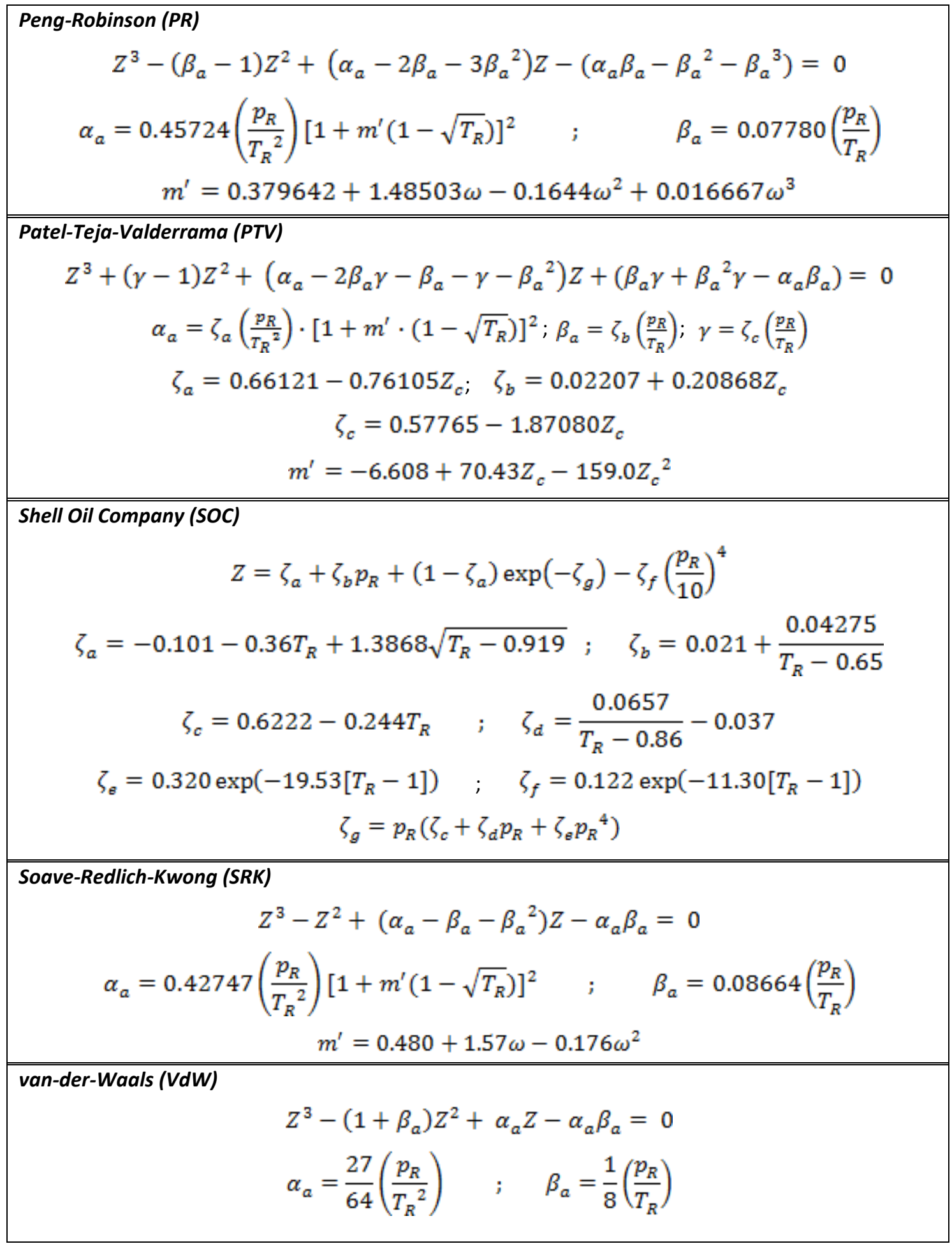

2.4Thermodynamic relations of a perfect gas

The thermodynamic relations listed in Table 2 are valid for ideal or perfect gases. These are widely known in the study of such gases, and have been used in the simulations devised in this work (Borges, 2009b).

\section{Natural gas}

\subsection{Typical chemical composition}

As described above, the main component of natural gas is methane, making up over $70 \%$ of the gas composition. Heavier hydrocarbons, such as ethane, propane, and butane, and small percentages of other constituents such as nitrogen 
Table 2. Thermodynamic properties of an ideal gas.

\begin{tabular}{lll}
\hline Equation of state & $\rho=\frac{p}{R T}$ & (11) \\
\hline Density & $d=\frac{M}{M a r}$ & (12) \\
\hline Specific heats & $\begin{array}{l}c_{p}-c_{V}=R \\
\frac{c_{p}}{c_{V}}=k\end{array}$ \\
\hline Enthalpy & $d h=c_{p} d T$ & $(13)$ \\
\hline Internal energy & $d u=c_{V} d T$ \\
\hline Entropy & $T d s=d u+p d v$ & $(14)$ \\
\hline
\end{tabular}

Table 3. The chemical composition and other properties of natural gas.

\begin{tabular}{cccccccc}
\hline Component & $\begin{array}{c}\mathrm{M} \\
{[\mathrm{kg} / \mathrm{kmol}]}\end{array}$ & $\begin{array}{c}T_{c} \\
{[\mathrm{~K}]}\end{array}$ & $\begin{array}{c}p_{c} \\
{[\mathrm{MPa}]}\end{array}$ & $Z_{c}$ & $\omega$ & $\begin{array}{c}c_{p} \\
{[\mathrm{~J} / \mathrm{kg} . \mathrm{K}]}\end{array}$ & $\begin{array}{c}c_{\mathrm{v}} \\
{[\mathrm{J} / \mathrm{kg} . \mathrm{K}]}\end{array}$ \\
\hline $\mathrm{CH}_{4}$ & 16.043 & 190.6 & 4.596 & 0.286 & 0.011 & 2253.3 & 1735.1 \\
$\mathrm{C}_{2} \mathrm{H}_{6}$ & 30.069 & 305.4 & 4.883 & 0.285 & 0.099 & 1754.3 & 1477.8 \\
$\mathrm{C}_{3} \mathrm{H}_{8}$ & 44.096 & 369.8 & 4.250 & 0.281 & 0.153 & 1672.9 & 1484.4 \\
$\mathrm{n}-\mathrm{C}_{4} \mathrm{H}_{10}$ & 58.123 & 425.2 & 3.796 & 0.274 & 0.199 & 1708.9 & 1565.9 \\
$\mathrm{i}-\mathrm{C}_{4} \mathrm{H}_{10}$ & 58.123 & 408.2 & 3.648 & 0.283 & 0.183 & 1673.5 & 1530.5 \\
$\mathrm{n}-\mathrm{C}_{5} \mathrm{H}_{12}$ & 72.151 & 469.7 & 3.370 & 0.262 & 0.251 & 1649.1 & 1533.9 \\
$\mathrm{i}-\mathrm{C}_{5} \mathrm{H}_{12}$ & 72.151 & 460.4 & 3.380 & 0.270 & 0.227 & 1645.0 & 1529.8 \\
$\mathrm{n}-\mathrm{C}_{6} \mathrm{H}_{14}$ & 86.178 & 507.5 & 3.010 & 0.264 & 0.299 & 1673.1 & 1576.6 \\
$\mathrm{~N}_{2}$ & 28.013 & 126.3 & 3.400 & 0.289 & 0.039 & 1037.5 & 740.71 \\
$\mathrm{O}_{2}$ & 32.050 & 154.8 & 5.043 & 0.287 & 0.025 & 917.00 & 657.59 \\
$\mathrm{CO}_{2}$ & 44.010 & 304.2 & 7.382 & 0.277 & 0.239 & 869.34 & 680.43 \\
\hline
\end{tabular}

and carbon dioxide complete the composition. In general, however, the composition of natural gas can vary widely, depending on the parameters of the production process, conditioning, processing, and transportation. In Brazil, the specification of natural gas for trade and transport is defined in the Resolution No. 16 , of June $17^{\text {th }} 2008$, proposed by ANP (the National Petroleum Agency). In general, however, its density is less than one and its gross calorific value ranges between 8000 and 10000 $\mathrm{kcal} / \mathrm{m}^{3}$. The properties of the natural gas mixture are required as input variables of the cubic equations of state. Table 3 shows the properties of the main pure substances that are more frequently used to characterize a given gas sample (Almeida \& Velasquez, 2013). These have been taken as a reference in the computational code developed in this work.

\section{RESULTS AND DISCUSSIONS}

\subsection{The computer program}

The results involve many calculations and evaluations of partial intermediate variables, which are obtained easily and quickly from a computational implementation. For this purpose, the experiment used the commercial software Matlab that is considered easy to handle and provides a friendly graphical interface. The input parameters basically correspond to the percentage of each individual component of natural gas. The program also allows the user to choose from a selection of options as far as the equations of state are considered. In this context, the user has two other options to choose from with regard to input temperature and pressure, as follows: (a) enter individual values for pressure and temperature; or (b) set up input values in a vector format for pressures and temperatures in the same 
Table 4. Results of a generic simulation performed with Matlab.

\begin{tabular}{l} 
*** General and thermodynamic properties of the natural gas $* * *$ \\
\hline Chemical composition of natural gas analyzed: \\
\hline CH4: 89.01 \\
C2H6: 5.93 \\
C3H8: 1.85 \\
nC4H10: 0.42 \\
iC4H10: 0.31 \\
nC5H12: 0.11 \\
iC5H12: 0.08 \\
nC6H14: 0.08 \\
O2: 0 \\
N2: 0.67 \\
CO2: 1.57 \\
\hline PARTIAL RESULTS - PROPERTIES OF NATURAL GAS: \\
\hline molecular weight: $18.3877 \mathrm{~kg} / \mathrm{kmol}$ \\
density: 0.63485 \\
constant gas: $452.1498 \mathrm{~J} / \mathrm{kmol} \mathrm{K}$ \\
critical temperature: $204.569 \mathrm{~K}$ \\
critical pressure:4.6338MPa \\
critical compressibility factor: 0.2857 \\
acentric factor: 0.024606 \\
specific heat at constant pressure: $2178.1013 \mathrm{~J} / \mathrm{kmol} \mathrm{K}$ \\
specific heat at constant volume: $1724.6467 \mathrm{~J} / \mathrm{kmol} \mathrm{K}$ \\
\hline Calculation of Z and dynamic viscosity \\
reference pressure - MPa: 0.7 \\
state equation selected: Soave-Redlich-Kwong \\
\hline FINAL RESULTS - PROPERTIES OF NATURAL GAS: \\
specific mass: $0.005282 \mathrm{~g} / \mathrm{cm} 3$ \\
compressibility factor: 0.98383 \\
\hline
\end{tabular}

simulation. Finally, one can obtain the complete list of values that correspond to various physical and thermodynamic properties of natural gas, based on the chemical composition previously defined as a program input parameter within the range of pressure and temperature (or any given individual value) chosen for each simulation. In the case of the compressibility factor, different results can be obtained, depending on the selected equation of state, in which case one must carry out comparative analyses from the available options.

Table 4 illustrates the "script" results from a simulation performed with the average chemical composition of natural gas distributed by COMPAGAS with temperature and pressure of 7 bar $(0.7 \mathrm{MPa})$ and $20{ }^{\circ} \mathrm{C}$, respectively, and adopting, by choice, the equation state of SoaveRedlich-Kwong (SRK).

\subsection{Comparative evaluation with field operational data}

To validate and compare the results obtained by this proposal, a number of real values of compressibility factor was recorded for samples of the COMPAGAS distribution network. COMPAGAS currently has a distribution network covering pipes in carbon steel and HDPE, with a length of approximately $600 \mathrm{~km}$, operating with distribution pressures between 4 and 35 bar. Also, because the city of Curitiba and its metropolitan region have a very sharp temperature variation throughout the year, field measurements were carried out over a longer period of time, aiming at contemplating such variability. 


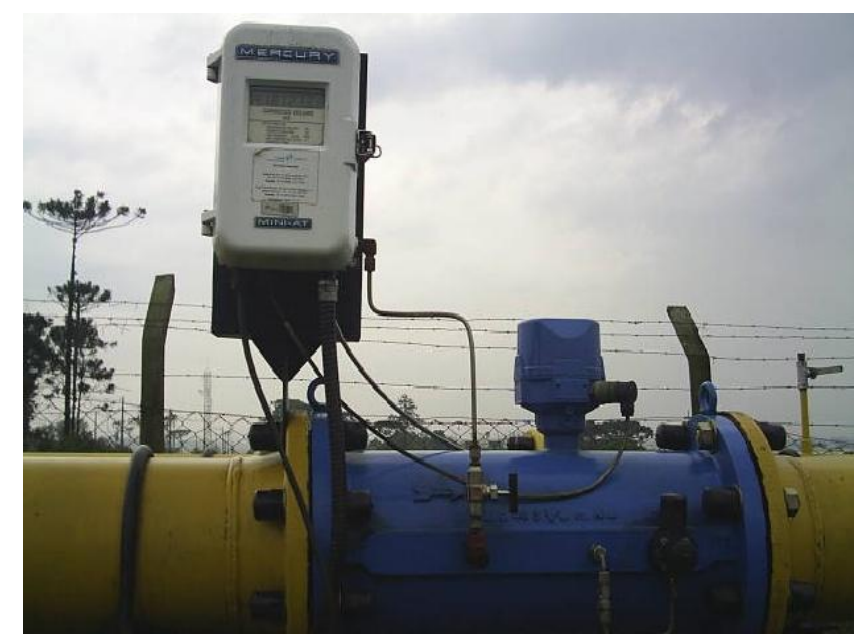

Figure 2. Standard Electrocorretor (COMPAGAS).

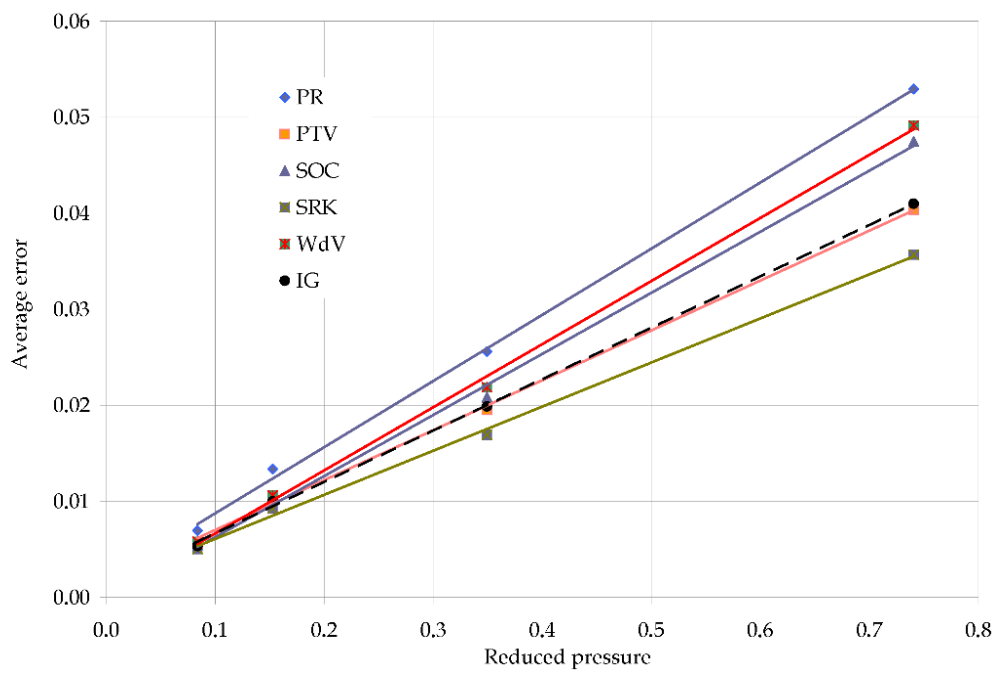

Figure 3. Variations on the average error $\bar{\varepsilon}$ as a function of reduced pressure.

Figure 2 illustrates the device used to acquire field data. It is an electrocorretor - a device programmed to carry out the calculation of the mass consumption of fluid from the measured values of volumetric consumption, as a function of pressure and temperature (Almeida \& Velasquez, 2013). This device is also programmed to calculate instantly the gas compressibility factor by using the equation of state of the AGA-8 standard (1994), considered one of the most accurate and conventionally adopted by most distributors of natural gas in Brazil.

Finally, Equation (18) was used to evaluate the existing average error on the operational values for each nominal relative pressure obtained by the proposed models described in Table 1 , including the ideal gas model (IG):

$\bar{\varepsilon}=\frac{1}{n} \sum_{i=1}^{n} \sqrt{\left(Z_{i}-Z_{A G A}\right)^{2}}$

Figure 3 illustrates the results obtained, representing the average error as a function of reduced pressure obtained by averaging each set of measured data. For the present case, the results show that the equation of state of Soave-RedlichKwong (SRK) provides the lowest errors in relation to the AGA-8 equation. One can also observe that the errors were obtained within an acceptable tolerance, confirming the feasibility regarding the use of this proposal. 


\section{CONCLUSIONS}

In the preliminary calculations of a project for natural gas operations, the proposal developed in this work allowed to assess thermodynamic properties of natural gas with reasonable and sufficient accuracy, from its pressure, temperature, and composition standpoints. In parallel, using Matlab, simple simulations were made to show the numeric capacity of this tool, and the results encourage their application in other various engineering applications. Also, given the amount of equations of state available in bibliographic reference for determining the compressibility factor of natural gas, comparative options were examined for some of these equations, giving the users the possibility of selecting the most representative model for their problems.

Finally, a comparison of the numerical data obtained from the simulations generated with data from the actual operating conditions of a gas pipeline distribution network confirmed and validated the developed model, based on the low error levels obtained.

\section{NOMENCLATURE}

$$
\begin{aligned}
& \alpha_{a^{\nu}} \boldsymbol{\beta}_{a^{\nu}} \boldsymbol{\gamma} \rightarrow \text { parameters of the equations of state } \\
& \bar{\varepsilon} \rightarrow \text { average error } \\
& \zeta_{a^{, \ldots x}} \zeta_{g} \rightarrow \text { parameters of the equations of state } \\
& \text { Patel-Teja-Valderrama and Soave- } \\
& \text { Redlich-Kwong } \\
& \boldsymbol{\mu} \quad \rightarrow \text { dynamic viscosity } \\
& \rho \quad \rightarrow \text { specific mass } \\
& \boldsymbol{\tau}_{W} \quad \rightarrow \text { shear stress } \\
& v \quad \rightarrow \text { specific volume } \\
& v_{c} \quad \rightarrow \text { critical specific volume } \\
& v_{R} \quad \rightarrow \text { reduced specific volume } \\
& \omega \rightarrow \text { acentric factor } \\
& c_{p}, c_{V} \rightarrow \text { specific heat at constant pressure and } \\
& \text { specific heat at constant volume } \\
& \text { d } \quad \rightarrow \text { gas density } \\
& h \quad \rightarrow \text { specific enthalpy per unit mass } \\
& k \quad \rightarrow \text { ratio of specific heats } \\
& m^{\theta} \quad \rightarrow \text { parameter of the equations of state }
\end{aligned}
$$

\begin{tabular}{|c|c|}
\hline M & $\rightarrow$ molar mass of the gas \\
\hline$M_{a r}$ & $\rightarrow$ molar mass of air \\
\hline$p$ & $\rightarrow$ pressure \\
\hline$p_{c}$ & $\rightarrow$ critical pressure \\
\hline$p_{p c}$ & $\rightarrow$ pseudocritical pressure \\
\hline$p_{R}$ & $\rightarrow$ reduced pressure \\
\hline$R$ & $\rightarrow$ gas constant $\left(R=R_{u} / M\right)$ \\
\hline$R_{u}$ & $\rightarrow$ universal gas constant \\
\hline$s$ & $\rightarrow$ entropy per unit mass \\
\hline$T$ & $\rightarrow$ absolute temperature \\
\hline$T_{c}$ & $\rightarrow$ critical temperature \\
\hline$T_{p c}$ & $\rightarrow$ pseudocritical temperature \\
\hline$T_{R}$ & $\rightarrow$ reduced temperature \\
\hline$u$ & $\rightarrow$ specific internal energy per unit mass \\
\hline$v$ & $\rightarrow$ speed \\
\hline$X, Y$ & $\begin{array}{l}\rightarrow \text { empirical factors in the dynamic } \\
\text { viscosity equation }\end{array}$ \\
\hline Z & $\rightarrow$ compressibility factor \\
\hline$Z_{c}$ & $\rightarrow$ critical compressibility factor \\
\hline$Z_{\mathfrak{i}}$ & $\rightarrow$ calculated compressibility factor \\
\hline$Z_{A G A}$ & $\rightarrow$ compressibility factor measured \\
\hline
\end{tabular}

\section{REFERENCES}

AGA REPORT 8 - Compressibility factors of natural gas and other hydrocarbon gases. EUA: American Gas Association, Virginia, 1994.

Almeida, J. C.; Velasquez, J. A. A.; Barbieri, R. A methodology for calculating the natural gas compressibility factor for a distribution network. Petroleum Science and Technology, v. 32, p. 26162624, 2013.

http://dx.doi.org/10.1080/10916466.2012.755194

Borges, E. M. L. Avaliação de correlações e equações de estado para determinação de fatores de compressibilidade de gás natural. Dissertação de Mestrado do Programa de Pós-Graduação em Engenharia Química da Universidade do Estado do Rio de Janeiro - UERJ, Rio de Janeiro, Brasil, 2009a (in Portuguese). 
Borges, P. R. Propriedades termodinâmicas do gás natural. Gerência Executiva de Abastecimento e Logística da Petrobrás. Documento AB-LO 08/2009, 2009b (in Portuguese).

Chvidchenko V. Estudo comparativo do desempenho das equações de estado CPA, SRK e PR na modelagem de sistemas de interesse para a indústria do gás natural. Dissertação de Mestrado em Tecnologia de Processos Químicos e Bioquímicos. Escola de Química da Universidade Federal do Rio de Janeiro, Rio de Janeiro, Brasil, 2008 (in Portuguese).

Kay, W.B. Density of hydrocarbon gas and vapors at high temperature and pressures. Industrial Engineering Chemistry, v. 28, p. 10141019, 1936.
Kumar N., Compressibility factors for natural and sour reservoir gases by correlations and cubic equations of state. Master's thesis in petroleum engineering. Faculty of Texas Tech University, EUA, 2004.

Peixoto, J. G. Química-Física - Tabelas gerais. Universidade do Minho, DEB, 2005/2006 (in portuguese).

Rabah, A. A.; Mohamed, S. A. A new three parameter cubic equation of state. Transactions $\mathbf{C}$ : Chemistry and Chemical Engineering. v. 17(2), p. 177-184, 2010. 